\title{
A forced running wheel system with a microcontroller that provides high-intensity exercise training in an animal ischemic stroke model
}

\author{
C.C. Chen ${ }^{1}$, M.W. Chang ${ }^{2}$, C.P. Chang ${ }^{3}$, S.C. Chan ${ }^{1}$, W.Y. Chang ${ }^{1}$, C.L. Yang ${ }^{1}$ and M.T. Lin ${ }^{4}$ \\ ${ }^{1}$ Department of Electrical Engineering, National Cheng-Kung University, Tainan, Taiwan \\ ${ }^{2}$ Department of Electrical Engineering, Southern Taiwan University of Science and Technology, Tainan, Taiwan \\ ${ }^{3}$ Department of Biotechnology, Southern Taiwan University of Science and Technology, Tainan, Taiwan \\ ${ }^{4}$ Department of Medical Research, Chi Mei Medical Center, Tainan, Taiwan
}

\begin{abstract}
We developed a forced non-electric-shock running wheel (FNESRW) system that provides rats with high-intensity exercise training using automatic exercise training patterns that are controlled by a microcontroller. The proposed system successfully makes a breakthrough in the traditional motorized running wheel to allow rats to perform high-intensity training and to enable comparisons with the treadmill at the same exercise intensity without any electric shock. A polyvinyl chloride runway with a rough rubber surface was coated on the periphery of the wheel so as to permit automatic acceleration training, and which allowed the rats to run consistently at high speeds $(30 \mathrm{~m} / \mathrm{min}$ for $1 \mathrm{~h})$. An animal ischemic stroke model was used to validate the proposed system. FNESRW, treadmill, control, and sham groups were studied. The FNESRW and treadmill groups underwent 3 weeks of endurance running training. After 3 weeks, the experiments of middle cerebral artery occlusion, the modified neurological severity score (mNSS), an inclined plane test, and triphenyltetrazolium chloride were performed to evaluate the effectiveness of the proposed platform. The proposed platform showed that enhancement of motor function, mNSS, and infarct volumes was significantly stronger in the FNESRW group than the control group $(P<0.05)$ and similar to the treadmill group. The experimental data demonstrated that the proposed platform can be applied to test the benefit of exercisepreconditioning-induced neuroprotection using the animal stroke model. Additional advantages of the FNESRW system include stand-alone capability, independence of subjective human adjustment, and ease of use.
\end{abstract}

Key words: Forced non-electric-shock running wheel; Middle cerebral artery occlusion; Inclined plane test; Modified neurological severity score; Triphenyltetrazolium chloride

\section{Introduction}

Regular exercise is considered to be beneficial for the overall health of an organism in its normal and diseased states (1-3). An increasing number of studies have shown the beneficial effects of exercise on stroke-induced brain injuries in animal models $(4,5)$. The neuroprotective mechanisms of exercise in humans are the same as those in animals; therefore, animal injury models are used to verify the efficacy of exercise in humans (6-9). Rats are often used in these experiments. Six weeks of free-running wheel exercise enhances the survival of neural progenitor cells after a hemorrhagic stroke (6), and pretraining exercises on a free-running wheel for 7 weeks can stimulate neurogenesis in damaged neuronal cells (7). Additionally, 2 or 4 weeks of pretraining exercise on a treadmill reduces brain damage after ischemic stroke (8). Research has shown an exercise preconditioning benefit on neurological deficits and the infarct volume in rats that exercised on a treadmill at $15 \mathrm{~m} / \mathrm{min}$ for $30 \mathrm{~min} /$ day for 3 weeks and were then subjected to transient middle cerebral artery occlusion (MCAo) (9). However, more animal experiments used $20-30 \mathrm{~m} / \mathrm{min}$ as exercise preconditioning for the prevention of stroke than low-intensity training $(15 \mathrm{~m} / \mathrm{min})(4,10-13)$. These findings suggest that exercise preconditioning can produce neuroprotection from ischemic stroke and that this protection is manifested during reperfusion. In this study, an animal ischemic stroke model was triggered to validate the proposed system.

Correspondence: C.L. Yang, Department of Electrical Engineering, National Cheng-Kung University, No. 1, University Road, EE Dep., NCKU, Tainan 70101, Taiwan. Fax: +886-6-234-5482. E-mail: cyang@mail.ncku.edu.tw 
Animal locomotion platforms can be classified into voluntary running wheel and forced treadmill groups (14). The treadmill uses electric shock to force rats to run, whereas the running wheel allows voluntary running. Voluntary running is used to simulate daily human activities (1). In contrast, rats on a treadmill are forced to move for 0.5-1 h for 5-7 days per week, which simulates an athlete performing long-term exercise in a gym (15). Clinical anatomical experiments have found that these two types of exercise can enhance and maintain the energizing brain glucose transporter (GLUT-1 and GLUT-3) (16). Forced and voluntary exercise differentially affect neurophysiological performance $(17,18)$. Forced exercise effectively induces neuroprotection in stroke, but this is not the case with voluntary exercise (17). However, the voluntary activity leads to more prominent plastic changes in the hippocampal formation of rats than the forced exercise (18). The ability to control and maintain a high training intensity $(>20 \mathrm{~m} / \mathrm{min})$ is a major advantage of a treadmill. The disadvantages of a voluntary running wheel include a substantially lower intensity of exercise and more variation in the amount of exercise that each animal receives. However, two methods interfere with the experimental results from forced treadmills: 1) aversive electric shocks to force exercise training, and 2) manually adjusting the speed of the exercise training at the beginning of the exercise and during the training stage. In the anatomical experimental data, when training with electric shocks, it is difficult to distinguish whether psychological stress or exercise training induces a physiological phenomenon. In addition, psychological stress may have negative effects, including adrenal hypertrophy, splenic atrophy, and increased circulating corticosterone $(9,17,19)$. These extra physiological responses interfere with the experimental results. Therefore, this study proposes a forced nonelectric-shock running wheel (FNESRW) exercise platform to train animals maintaining high-intensive training and to minimize the influence of psychological stress. Moreover, an automated exercise-training model is included to accurately control the amount of exercise activity and to reduce the disadvantages of manual training on treadmills.

Motorized running wheels (MRWs) are commercially available for researchers (20-22). However, Leasure and Jones (20) found that rats were reluctant to run at speeds higher than $20 \mathrm{~m} / \mathrm{min}$ on the currently available MRWs. These MRWs are currently used in research for low-speed running (20-23), and the lack of smooth automation training patterns allows rats to fall and become injured during highintensity training. High-intensity exercise training (>20 m/ min) triggers effective neurophysiological protection and recovery in a more effective and faster manner than low-intensity training for some clinical end-points (24-27). Therefore, this study sought to overcome the difficulties of high-intensity exercise training on a traditional MRW and excluded the interference effects of the treadmill with electric shock that is applied to an animal model. We developed an

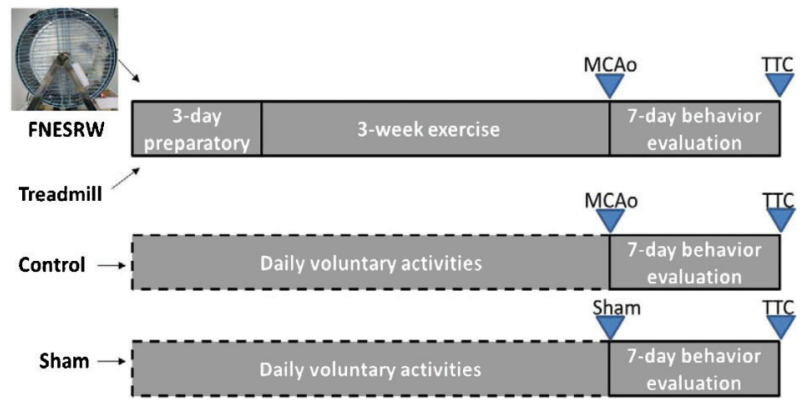

Figure 1. Timeline of the experimental design. Only the forced non-electric-shock running wheel (FNESRW) and treadmill groups followed 3 weeks of running training, and only the sham group was not triggered by middle cerebral artery occlusion (MCAo). All animals were observed for 7 days using a behavior test.

automatic and progressive exercise training pattern that spans from preparatory training to official exercise running, prevents injuries, and removes the disadvantages of a human operator. Moreover, a polyvinyl chloride (PVC) runway with a rough rubber surface was coated onto the periphery of the wheel to allow the rats to run smoothly and consistently at high speeds ( $30 \mathrm{~m} / \mathrm{min}$ for $1 \mathrm{~h}$ ). The firmware of the system can be easily modified to suit movement patterns for other applications. We describe a fully functional prototype of the system and its implementation. The results of this study show that the infarction volume can be reduced through preconditioning exercise with the proposed platform.

\section{Material and Methods}

The proposed FNESRW with an automatic training function can train animals to perform high-intensity running while avoiding the use of electric shocks, which allows the impact of exercise on the infarct volume in cerebral ischemic stroke to be verified without interference. The animals used in the experiment were randomly assigned to four groups (FNESRW, treadmill, sham, and control). Only the FNESRW and treadmill groups followed a 3-week exercise training program (Figure 1). Exercise speed training data from the 3-day preparatory training were acquired using the manual mode of the proposed running wheel exercise platform, which was used to construct a complete set of automatic exercise training models for the subsequent 3 weeks of exercise. After the 3 weeks of exercise, a stroke was induced in the FNESRW, treadmill, and control animals with 60 min of MCAo, which was followed by 7-day reperfusion using an intraluminal filament. The same surgery was performed for the sham group but without triggering blocked arteries. To evaluate the damage and recovery from the stroke, an inclined plane device test (28) and the modified neurological severity score (mNSS) (29) were used to evaluate neurological damage for the next 7 days. Finally, the animals were killed 


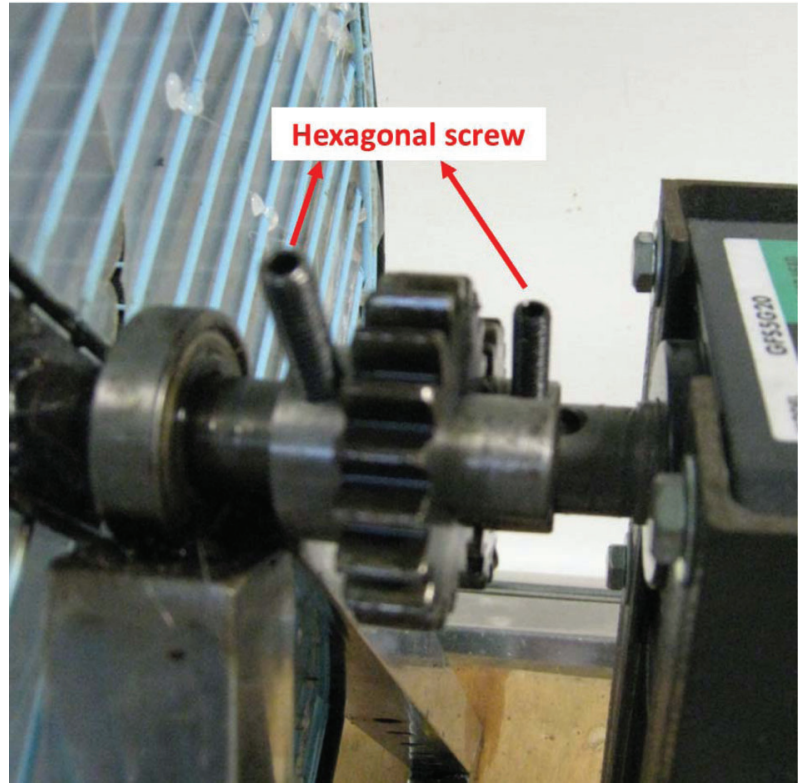

Figure 2. Center axis of the motor and roller are embedded by gears. A hexagonal screw is used to fasten the assembly.

by exposure to triphenyltetrazolium chloride (TTC) to assess infarct volume.

\section{FNESRW}

The proposed microcontroller-based FNESRW system facilitates exercise preconditioning for small laboratory animals such as rats. The FNESRW system consists of four parts: 1) a roller platform coated with a PVC runway the sides of the center axis of the wheel are welded to the triangular metal framework to support the commercially available roller that is constructed of metal bars, and the bearing is embedded in the center of the wheel to enable free rotation and create a voluntary running wheel configuration; 2) a high-torque, brushless direct current (DC) motor, which drives the roller - a hexagonal screw latches to a gear into the central axis of the motor and wheel, and the gears on the motor and the wheel are the same size, and these two gears are mutually engaged and can be removed (Figure 2) to create a forced running wheel configuration; 3 ) a flexible microcontroller is used to precisely control the motor speed by using pulse-width modulation (PWM) for the automatic progressive training function; and 4) a conventional computer with a parallel port is used to connect the system to a computer, which writes and loads the firmware that is used in the microcontroller and controls the speed of the FNESRW system.

A photograph of the lab-built apparatus is shown in Figure 3 . The running platform has a wheel that is $35 \mathrm{~cm}$ in diameter and $15 \mathrm{~cm}$ wide, and the height from the triangle bracket to the bottom of the wheel is $20 \mathrm{~cm}$. The outer ring of the wheel is coated with a PVC runway with a

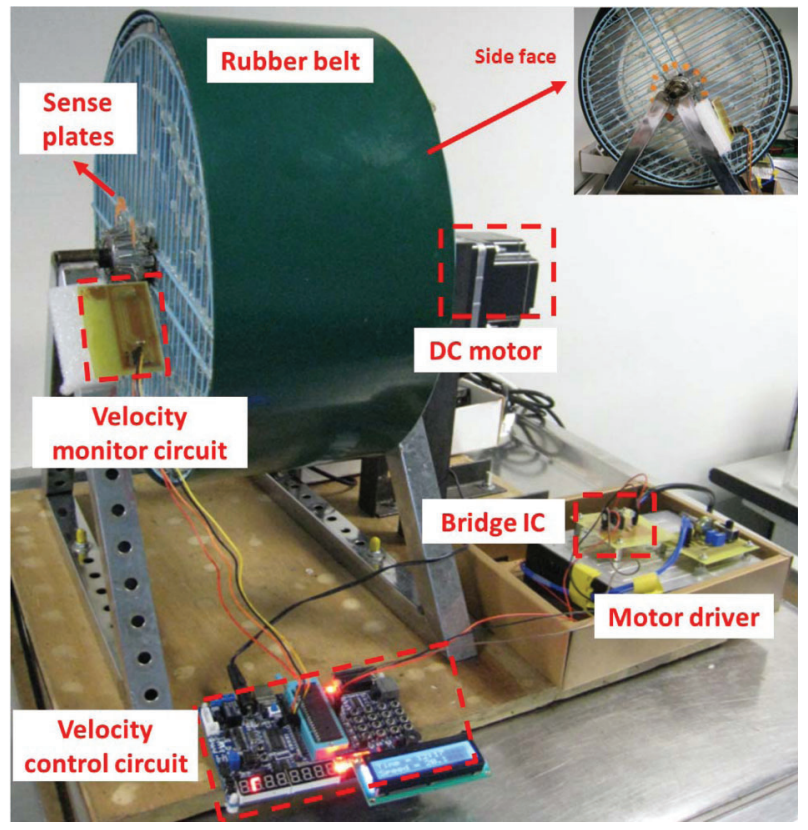

Figure 3. Forced non-electric-shock running wheel (FNESRW).

rough surface, which is tightly fixed to the outside of the wheel frame with strong Velcro tape, making it removable. Most rats fail to run up and do not fully step on the bars at $20 \mathrm{~m} / \mathrm{min}$ on the running wheel without the PVC runway (the structure of the commercially available running wheel). This design is effective for overcoming the highintensity training restrictions of commercially available MRWs, thus allowing rats to run relatively stably and preventing injuries at high speeds $(20 \mathrm{~m} / \mathrm{min}$ or more). Eleven sensing plates were placed on the wheel rim to detect rotation speed (Figure 3 ).

The speed of the FNESRW is controlled by a DC motor and its driver. The system can be switched between manual and automatic modes using a jumper switch. The manual mode employs a variable resistor to produce various voltages and the control speed, whereas the automatic mode is controlled by the microcontroller firmware with PWM. Six automatic training speed patterns exist: three for preparatory training and three for formal training.

A block diagram of the control unit hardware is shown in Figure 4. The control unit consists of a microcontroller, a liquid-crystal display (LCD), a touch-button keypad, an insystem programing interface, a velocity monitor, a velocity control, a jumper switch, and a power supply (a brief description of each component is provided below).

Microcontroller. The system is based on an AT89S52 microcontroller (ATMEL Corp., USA) that provides a highly flexible and cost-effective solution for embedded control applications. The AT89S52 includes four programmable ports, two external interrupt sources, three timers or counters, one programmable serial USART, $8 \mathrm{kB}$ of program 


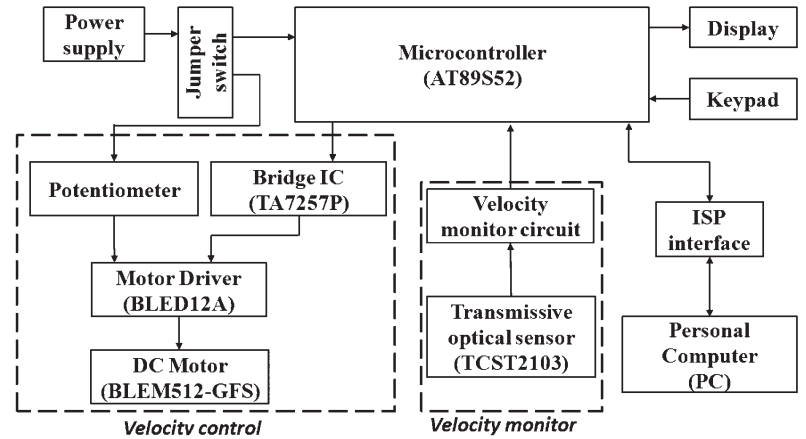

Figure 4. Block diagram of the microcontroller-based control unit. IC: integrated circuit.

memory, and 256 B of RAM for data.

Display. A conventional LCD with $2 \times 10$ characters shows the rotation speed of the proposed running wheel to the local user. The driver and built-in backlight are included with this off-the-shelf LCD module.

Keypad. A $4 \times 4$ button switch is used for the user interface. The six buttons were assigned three commands each for preparatory and formal trainings. Therefore, the user can input commands that trigger the automatic training pattern. These instructions can also be input using the keyboard of an attached PC.

Velocity monitor. The rotation of the proposed running wheel was detected using 11 sensing plates and a transmissive optical sensor (TCRT2103, Vishay Semiconductors, USA). A velocity-monitoring circuit was designed to process the received signals, which were sent to the microcontroller for speed calculation before display.

Velocity control. The speed control was set to manual or automatic mode using a jumper switch. The automatic mode provided a consistent and precise procedure for training rats and eliminated the human factor. The manual mode allowed the training to be dynamically controlled by a variable resistor (potentiometer). The automatic mode contained six training models that were triggered by the six buttons. The velocity control function of automatic training was achieved using a microcontroller (AT89S52), a bridge integrated circuit (IC; TA7257P, Toshiba, Japan), a motor driver (BLED12A, Oriental Motor, Japan), and a DC motor (BLEM512-GFS, Oriental Motor). The motor torque was $8.6 \mathrm{~N} \cdot \mathrm{m}$, and it effectively drove the running wheel platform (approximately $1 \mathrm{~kg}$ ) in addition to the weight of an experimental rat (250 to $350 \mathrm{~g}$ ) with a sufficient margin for an instant reaction to the force that was created by a running rat. The pulse signal of the microcontroller generated a specific voltage through the bridge IC, which was used to control the motor driver. The bridge IC was a full-bridge driver that converted the logic input signal into an analog signal.

Power supply. A conventional linear power supply provided regulated DC voltage $(+5 \mathrm{~V})$ for all components of the system, except for the DC motor driver and DC motor, which were directly powered from the main city power.

\section{Automatic training model construction}

The progressive training model was constructed to allow the rats to run steadily and continuously at $30 \mathrm{~m} / \mathrm{min}$ for $1 \mathrm{~h}$. Because of the curved structure of the running wheel, it was more difficult for the rats to be trained to run quickly and smoothly at high speeds (20 m/min or more) compared with training on a traditional treadmill. When accelerating, rats could easily fall and become injured. Some rats could jump from high to low positions, and such running behavior was relatively more labor saving than the linear motion of the treadmill. Therefore, an automatic progressive exercise-training model was designed to enable the animals to smoothly accelerate from 0 to $30 \mathrm{~m} / \mathrm{min}$ and prevent the skipping of parts of the exercise on rollers. The acceleration curve was systematically established and repeated daily to remove human error. In this study, a manual, progressive training method was used to train the rats to reach and maintain $20 \mathrm{~m} / \mathrm{min}$ within 3 days. Animal movement experiments usually use a preparatory training period of 3 days to allow the rats to adapt and learn the $20 \mathrm{~m} / \mathrm{min}$ running training $(4,10,30)$. The rats are injured easily when running at speeds of $20 \mathrm{~m} / \mathrm{min}$ without the adaptive learning stage. Nine rats were used in the experiments to construct training patterns for 3 days (30 min/day) using the manual mode. The training data of the velocity for various times are shown in Table 1.

Table 1. Experimental training data from the first to the third day.

\begin{tabular}{|c|c|c|c|c|c|c|c|c|}
\hline & \multicolumn{7}{|c|}{ Training data (time vs velocity) } & \multirow{2}{*}{ Time up to $95 \%$ final velocity } \\
\hline \multicolumn{8}{|l|}{ 1st day } & \\
\hline Time (s) & 0 & 200 & 400 & 600 & 800 & 1000 & 1200 & $=600 \mathrm{~s}$ \\
\hline Velocity (m/min) & 8 & 11.1 & 12.5 & 12.7 & 12.8 & 12.9 & 13 & \\
\hline \multicolumn{9}{|l|}{ 2nd day } \\
\hline Time (s) & 0 & 100 & 200 & 300 & 400 & 500 & 600 & $=300 \mathrm{~s}$ \\
\hline Velocity (m/min) & 8 & 13.3 & 14.8 & 15.6 & 15.8 & 15.9 & 16 & \\
\hline \multicolumn{9}{|l|}{ 3rd day } \\
\hline Time (s) & 0 & 60 & 120 & 180 & 240 & 300 & 3600 & $=180 \mathrm{~s}$ \\
\hline Velocity (m/min) & 8 & 15.9 & 18.4 & 19.4 & 19.8 & 19.9 & 20 & \\
\hline
\end{tabular}




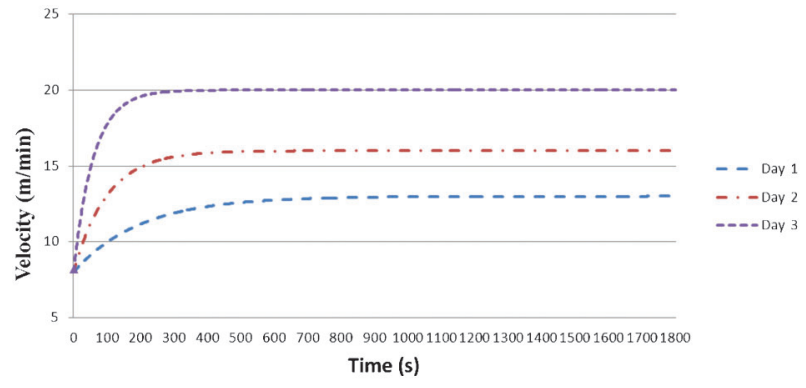

Figure 5. Construction of the three exponential exercise acceleration training model.

Automatic animal training patterns were constructed for rats without prior running wheel experience, and they learned to run at $20 \mathrm{~m} / \mathrm{min}$ (a medium-intensity exercise) within 3 days. Progressive acceleration was used to train the rats. The speed was temporarily reduced when a rat fell, and was raised when a rat ran steadily. The training speed was steadily increased, until the maximum speed that the rat could maintain while running smoothly was determined. On Day 1, the average maximum speed was approximately $13 \mathrm{~m} / \mathrm{min}$. The acceleration slope was gradually raised on Day 2 , and it was increasingly accelerated on Day 3 to achieve the desired maximum average speed of approximately $20 \mathrm{~m} / \mathrm{min}$. The rats reached higher speeds more quickly as they familiarized themselves with the running wheel. The daily maximum steady velocity showed an upward trend.

The accelerative curve showed a similar exponential distribution for the actual experimental training velocity data. Therefore, in this study, an exponential exercise acceleration training curve for 3 days was constructed (Figure 5), where the $x$-axis is time and the $y$-axis is the corresponding controlled velocity. The velocity at time $t$ is

velocity $(t)=A \times\left(1-\mathrm{e}^{-t / \tau}\right)+B$

where $A$ and $B$ are constants and $\tau$ is the time constant. After fitting the experimental data for the first day into the training model, A was set to 5 and $B$ was set to 8 . The acceleration slope of the velocity was obtained by changing the time constant $\tau$. Parameter construction of the training model for the other days followed the same method. The maximum training speed of the second week in the study was increased to $30 \mathrm{~m} / \mathrm{min}$ based on the exponential accelerative training model. The microcontroller firmware was programmed to change the output of PWM and to control the speed of the DC motor through the bridge IC

\section{Software}

Speed control and speed detection software were developed for the FNESRW system. First, the speed control software facilitated accurate and smooth acceleration control during the initial 3 days of preparatory training and 3 weeks of formal training. The goal of the 3-day preparatory training was to familiarize the rats with the roller and condition them to run smoothly at $20 \mathrm{~m} / \mathrm{min}$. The manual training data were analyzed to extract the speed-controllable parameters for an automated exponential training speed curve by PWM control. Second, speed detection software facilitated the recording of distance information using the microcontroller external interrupt (external INT0) service routine. The external INTO was triggered using the transmissive optical sensor to import data from the 11 sensing plates. Instantaneous speed was derived by dividing by the time and was displayed on the LCD.

The microcontroller firmware was written in the $C$ programming language and included the main program, two timer-interrupt service routines, and one externalinterrupt service routine. The main program consisted of a loop that calculated and displayed the training speed (Figure S1). The global variable "Num_Sensing" was the sensed number of the transmissive optical sensors; Num_Sensing was incremented as one unit per $10-\mathrm{cm}$ rotation, and the global variable "Time_0" was a second counter. Therefore, rotational speed was obtained as speed $=$ Num_Sensing $\times 10 /$ Time_0. The speed was displayed on the LCD, and the main program awaited commands from the local keypad. Commands A through $C$ were the preparatory training speed curves for the first, second, and third days, respectively. After INO, Timer 0 , and Timer 1 were enabled, a speed table of the training curve for the first to third days was created based on Equation 1. A PWM value was generated every $10 \mathrm{~s}$ according to the table that was created during the training period. Commands $D$ through $F$ corresponded to the first, second, and third weeks of the formal training curve, with maximum speeds and durations of $20 \mathrm{~m} / \mathrm{min}$ for $30 \mathrm{~min}$, $30 \mathrm{~m} / \mathrm{min}$ for $30 \mathrm{~min}$, and $30 \mathrm{~m} / \mathrm{min}$ for $60 \mathrm{~min}$, respectively. Two timer-interrupt service routines were used: Timer 0 and Timer 1 (Figure S2A and B, respectively). Timer 0 was used to calculate the time (in seconds) of a wheel rotation. Timer 1 was used to generate the corresponding PWM value for the time unit, which was used to update the PWM value every $10 \mathrm{~s}$ until the end of the exercise (Exer Time).

One external-interrupt service routine, INT0, was in edge-trigger mode for the transmissive sensing signal during wheel rotation (Figure S2C). The interrupt signal was triggered by the sensing action of the transmissive optical sensor, the interval between the figure sensing plates was $10 \mathrm{~cm}$, and running distance information was recorded there.

\section{Calibration}

A working prototype was implemented for the purpose of this study. First, the rotation speed was calibrated, and an accurate reference speed was measured using a speedometer (OXP-2A, Oriental Motor) and compared to 


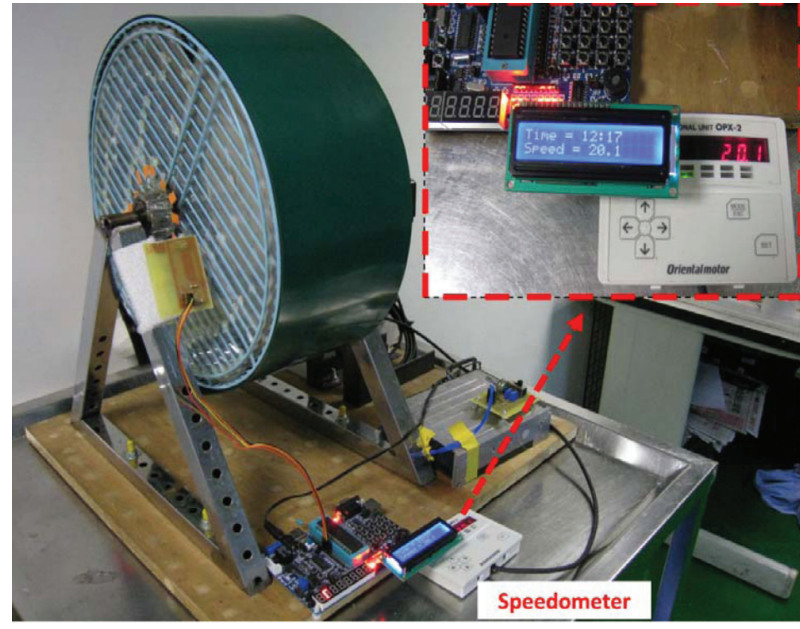

Figure 6. Speedometer used for speed calibration.

the speed monitor module for verification (the speedometer is shown in Figure 6). The manual and automatic modes of the proposed system were used to verify the speed of rotation and to compare the deviation between the speedometer and the speed monitor module. Manual mode was verified by randomly setting the speed and comparing the speedometer value to that of the speed monitor module. To verify automatic mode, one of the six keys on the keypad was pressed, and the speed value of the speedometer was compared to that of the speed monitor module. The speedometer data were accessed using the microcontroller through the speed-out connection port of the motor driver (BLED12A, Oriental Motor), which generated 600 pulse signals per cycle. The resulting errors were in the range of $\pm 0.1 \mathrm{~m} / \mathrm{min}$.

\section{Experimental animals}

Adult male Wistar rats (average weight $228 \pm 22 \mathrm{~g}$ ) were obtained from BioLASCO Taiwan Co., Ltd (Taiwan). The animals were housed in groups of four at an ambient temperature of $21 \pm 1^{\circ} \mathrm{C}$, with a $12: 12$-h light-dark cycle. Pellet rat chow and tap water were available ad libitum. All protocols were approved by the Animal Ethics Committee of the Chi-Mei Medical Center (Tainan, Taiwan) in accordance with the Guide for the Care and Use of Laboratory Animals of the National Institutes of Health (USA) and the Guidelines of the Animal Welfare Act.

\section{Exercise training protocol}

Animals were randomly assigned to the FNESRW $(n=9)$, treadmill $(n=9)$, control $(n=9)$, or sham $(n=9)$ groups. The animals in the preconditioning exercise group were placed on the FNESRW and treadmill (Exer-6M Treadmill, Columbus Instruments, USA) for a 3-day preparatory training to reach the required, basic efficient exercise intensity of $20 \mathrm{~m} / \mathrm{min}$ for $30 \mathrm{~min}$. The animals were then subjected to official endurance running training 5 days a week for 3 weeks (running for $30 \mathrm{~min}$ at $20 \mathrm{~m} / \mathrm{min}, 30 \mathrm{~min}$ at $30 \mathrm{~m} / \mathrm{min}$, and $60 \mathrm{~min}$ at $30 \mathrm{~m} / \mathrm{min}$ at 1,2 , and 3 weeks of training, respectively). The work rate of the rats on this training protocol was approximately $70-75 \%$ of their maximal oxygen consumption (31), and the ambient temperature was controlled at $25 \pm 1^{\circ} \mathrm{C}$. Body weight and rectal temperature of the rats were recorded before and after running. The animals in the control and sham groups did not receive exercise training, but were allowed to run freely in their cages for 3 weeks.

\section{MCAo}

An MCAo was induced in exercised and nonexercised animals after 3 weeks according to the stroke model. Animals were anesthetized and maintained with 1\%-3\% halothane in $70 \% \mathrm{~N}_{2} \mathrm{O}$ and $30 \% \mathrm{O}_{2}$ with a face mask. The rectal temperature (colon temperature) was monitored throughout the surgical procedure and maintained at $36.5 \pm 0.5^{\circ} \mathrm{C}$ using a circulating heating pad. MCAo was induced with an intraluminal filament model (32) and maintained for $60 \mathrm{~min}$. In brief, the left common carotid, internal carotid artery (ICA), and external carotid artery (ECA) were exposed by surgical operation. A filament (a 4-0 nylon suture with a blunted tip, $0.37 \mathrm{~mm}$ in diameter; Doccol Corporation, USA) that was coated with poly-Llysine was inserted into the right ECA. Next, the direction of the suture was altered toward the ICA and gently advanced to occlude the MCAo. Reperfusion was established by withdrawal of the filament under anesthesia at the end of Day 7 . The reliability and effectiveness of this model to induce stroke was guaranteed using poly-L-lysine-coated intraluminal sutures, which yielded consistently large infarcts and greatly reduced variation in the results between different animals (33). Rats in the sham group underwent the same ECA and ICA procedures without the MCAo.

\section{mNSS}

The mNSS was used to measure various aspects of neurological function. The mNSS test is a composite of motor (muscle status and abnormal movement), sensory (visual, tactile, and proprioceptive), reflex (pinna, corneal, and startle), and balance tests (29). Neurological function is graded on a scale of 0 to 18 (normal and healthy score: 0 ; maximum deficit score: 18), where one point counts for the inability to perform the test or for the lack of a tested reflex; thus, a higher score represents more severe injury. All rats were able to perform the tests every day for 7 days after surgery.

\section{Inclined plane test}

The grab performance of all rats was evaluated using an inclined plane device that was developed in our laboratory (28), on each of the 7 days after surgery. The inclined plane test is a quantitative, objective, and sensitive method for evaluating motor deficits after cerebral ischemia 
in rats. It assesses the ability of the rat to prevent itself from falling over and determines the endurance strength in the upper and lower limbs. The sliding apparatus had a $60 \times 60 \mathrm{~cm}$ acrylic surface that could be inclined from $0^{\circ}$ (horizontal) to $70^{\circ}$. The floor of the enclosure was covered with hook-side Velcro fabric, which provided a secure surface for the rat to grab and hold. Each rat was initially placed on a $25^{\circ}$ inclined plane in the area of the hook-side Velcro fabric. The lower limbs were allowed to clutch the Velcro, and the upper limbs remained on the acrylic surface. The angle of the inclined plane was then raised until the lower limbs detached from the Velcro and the rat slid down. Once this occurred, the increase of the plane angle was stopped, and the angle was recorded. The inclined plane test was conducted for each rat once a day for 7 days.

\section{TTC staining and quantitative analysis of infarct volume}

After 7 days of behavioral evaluation, the infarct volume was analyzed. The rats were deeply anesthetized with urethane $(500 \mathrm{mg} / \mathrm{mL}, i p)$, and the brains were carefully removed and dissected into coronal, $1-\mathrm{mm}$ sections using a vibratome. The fresh brain slices were immersed in a $2 \%$ solution of 2-, 3-, and 5-TTC in normal saline at $37^{\circ} \mathrm{C}$ for $30 \mathrm{~min}$. The total infarct area was multiplied by the thickness of the brain sections to obtain the infarct volume. The areas of noninfarcted tissue ipsilateral and contralateral to the occluded side were measured using a scanner and an image analysis system (34). The noninfarcted area in the ipsilateral hemisphere was subtracted from that in the contralateral hemisphere, and the infarct volume was calculated using the following formula: corrected percentage of infarct volume $=$ (contralateral hemispheric volume minus ipsilateral noninfarcted volume)/contralateral hemispheric volume (35).

\section{Statistical analysis}

Data are reported as means $\pm \mathrm{SE}$. Based on the underlying assumption that the test data of each group satisfy Gaussian distribution, a parametric analysis was applied to compare the differences among the groups. Comparisons between the various groups were evaluated using a one-way analysis of variance, followed by the Fisher least-significant difference post hoc test. Pairwise comparison was performed using the Student $t$-test in the case of heterogeneity of variance of data. Data were analyzed using the Sigma Plot Software (USA), and, in all cases, statistical significance was set at $\mathrm{P}<0.05$.

\section{Results}

\section{mNSS}

The mNSS was used to assess the effects of exercise preconditioning (FNESRW and treadmill groups) on the
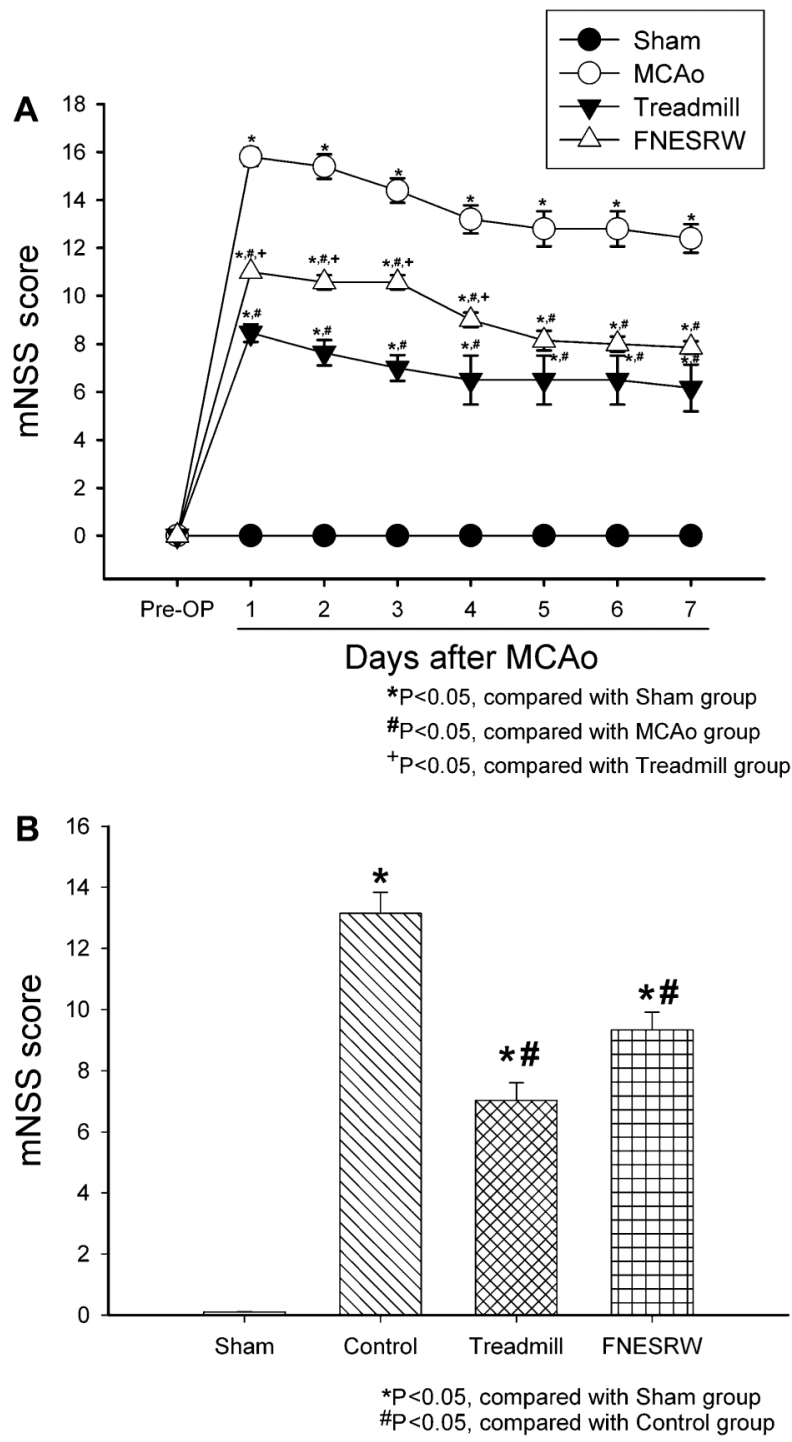

Figure 7. Average modified neurological severity score (mNSS) for the three groups over a period of 7 days. $A$, Daily average mNSS values. $B$, Average mNSS values for 7 days (means $\pm S D$ ). FNESRW: forced non-electric-shock running wheel; MCAo: middle cerebral artery occlusion. Analysis of variance showed a significant difference $(P<0.05)$, with an $F$-value of 139.532 for 3 and 32 degrees of freedom.

improvement of damaged neurological function after stroke. Figure 7 shows the average mNSS values of the four groups for the 7 days after surgery (means $\pm S D$ ). Scores of the sham group that were obtained before and after surgery were similar. The mNSS value in the control group was the highest (with heavy to moderate damage; Figure $7 A$ and $B$ ), and the mNSS values for the FNESRW group were significantly lower (moderate to minor damage) than those of the control group (Figure 7B; $P<0.05$ ). These results indicated that forced exercise preconditioning on 
the FNESRW was effective for stroke prevention. Although average scores for the treadmill were lower than those for the FNESRW, no significant difference was observed. Scores for the sham group were significantly lower than those for the FNESRW and treadmill groups (Figure 7B; $\mathrm{P}<0.05)$, which indicated the similar efficacy of nerve damage recovery over 7 days between the treadmill and FNESRW groups.

\section{Motor function}

Figure 8 shows the results of the motor performance of a lower limb grip that was measured using the inclined plane test for 7 days after surgery. Figure 8A shows the average inclined plane angle of the lower limb grip on each day for the four groups (sham, control, treadmill, and FNESRW), and Figure 8B shows the average angle that was obtained from the lower limb grip test for each group over 7 days (means $\pm S D$ ). The inclined plane angle of the control group was the lowest and significantly lower than that of the sham group on each of the 7 days (Figure $8 \mathrm{~A}$ and $B ; P<0.05)$. Although no significant difference between the inclined plane angles of the FNESRW and treadmill groups was observed (Figure 8B), the FNESRW group had significantly higher 7-day inclined plane angles than those of the control group (Figure 8B; $\mathrm{P}<0.05$ ). More specifically, the increased trend of inclined angles on each day for the FNESRW group was more obvious than that for the control group (Figure $8 \mathrm{~A}$ ), indicating that the recovery of motor function in the FNESRW group was faster than that in the control group.

\section{Infarct volume (TTC)}

The extent of the infarct lesions was examined using TTC staining (36). This method stains the normal parts of the brain red. The cerebral infarct regions in the brains of the MCAo model rats remained white after staining, and this reduced amount of staining clearly delineated the areas of infarction (Figure 9A). The experimental results show that the infarct size of the control group $\left(291 \pm 20 \mathrm{~mm}^{3}, \mathrm{P}<0.05\right)$ was significantly higher than that of the sham group after 7 days of reperfusion (Figure 9B). Infarct sizes of the treadmill $\left(42 \pm 88 \mathrm{~mm}^{3}, \mathrm{P}<0.05\right)$ and FNESRW $\left(104 \pm 22 \mathrm{~mm}^{3}, \mathrm{P}<0.05\right)$ groups were significantly lower than that of the control group (Figure 9B), indicating that endurance exercise training (either on a treadmill or FNESRW) can effectively reduce the infarct volume. There was no statistical difference between the treadmill and FNESRW groups in infarct area, indicating the similar benefit of exercise preconditioning in the animal ischemic stroke model.

\section{Discussion}

In this study, we developed an FNESRW platform with automatic exercise training patterns to force rats to run in high-intensive training, and the ischemic stroke model for
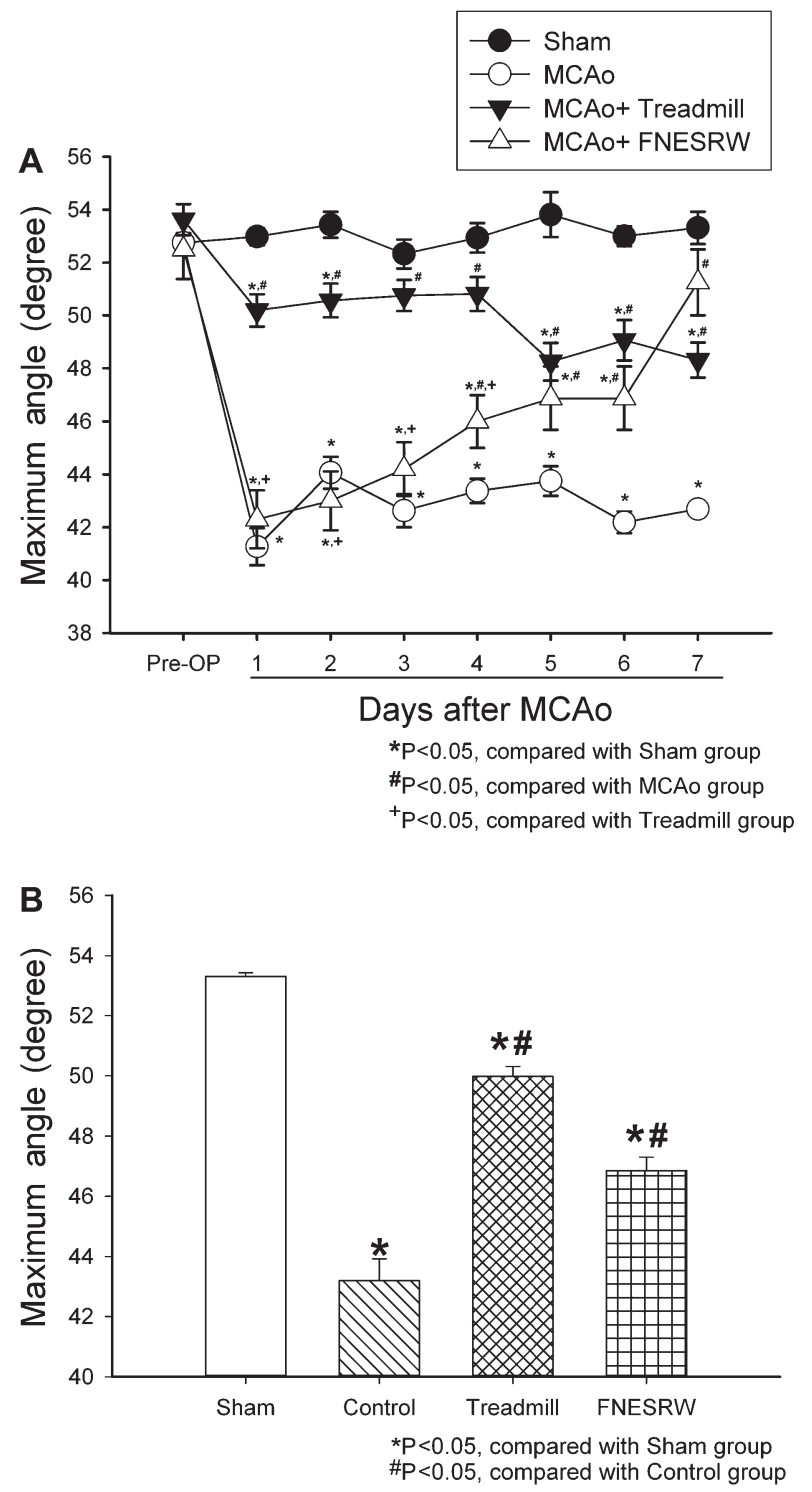

Figure 8. Average angle of the lower limb grip test over a period of 7 days. A, Average daily inclined plane angle. B, Average 7day inclined plane angle. Data are reported as means $\pm S D$. FNESRW: forced non-electric-shock running wheel; MCAo: middle cerebral artery occlusion. Analysis of variance showed a significant difference $(\mathrm{P}<0.05)$, with an $\mathrm{F}$-value of 25.315 for 3 and 32 degrees of freedom.

rats was used to verify the benefit of exercise training on the proposed system. During training, it was difficult for rats to exercise at $20 \mathrm{~m} / \mathrm{min}$, which is the required basic speed for effective exercise intensity (30), on the proposed platform without a rubber belt (the structure of the commercially available running wheel). Rats were forced to run at $20 \mathrm{~m} / \mathrm{min}$ or more on the structure of the MRW runway in our experiments, and the rats would clutch a cross bar and fall down, which induced injury, 

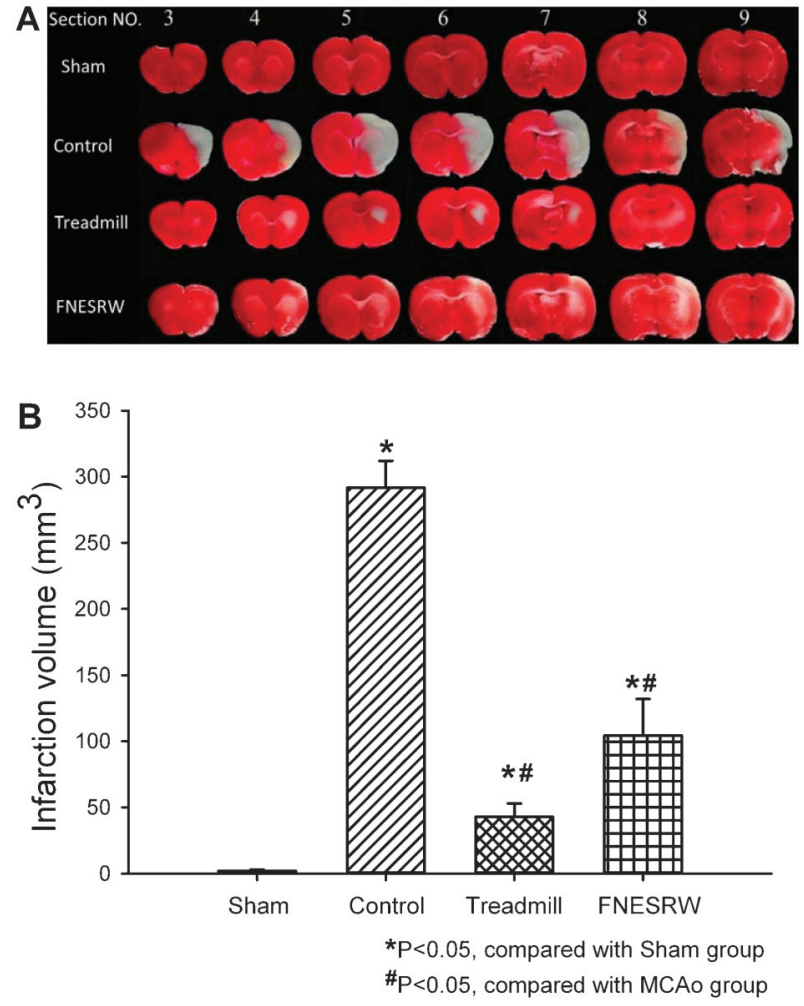

Figure 9. Cerebral infarction volume 7 days after reperfusion. $A$, Brain slices stained with TTC to visualize damaged lesions. $B$, Average of the lesion volumes that were assessed using the reduction in TTC staining (means \pm SD). FNESRW: forced nonelectric-shock running wheel; MCAo: middle cerebral artery occlusion. Analysis of variance showed a significant difference $(P<0.05)$, with an F-value of 107.872 for 3 and 32 degrees of freedom.

which is also a problem for commercially available MRWs (20). Two possible reasons exist for this occurrence. First, compared with the flat plane trajectory of a treadmill, the curved trajectory of a running wheel makes running more difficult and complex. Second, unlike the treadmill track belt, which rats can step onto easily and smoothly, gaps are present between the bars of the running wheel, which force the rats to run along the beams. To facilitate steady running at higher wheel speeds, a layer of PVC runway was coated onto the outside of the running wheel to prevent injury and improve running stability. This structure allowed the rats to perform steady, high-intensity training at $30 \mathrm{~m} / \mathrm{min}$ for $1 \mathrm{~h}$. Moreover, we automated the progressive exercisetraining model by gradually accelerating the training patterns to precisely and smoothly accelerate from 0 to $30 \mathrm{~m} / \mathrm{min}$, thus preventing the rats from falling and becoming injured.

The experimental results of the motor function (inclined plane test), mNSS values, and infarct volume for the FNESRW group were significantly stronger (Figures 7-9; $\mathrm{P}<0.05)$ compared with those of the control group and were similar to the treadmill group, which indicated that the FNESRW provided effective exercise training. Many animal neurophysiological studies have confirmed that the benefit of a forced treadmill is significantly higher than that of a voluntary running wheel, regardless of the intrinsic neuroprotection $(16,17,37)$ or strengthening of the muscles (38). The experimental data obtained in the present study indicated that the FNESRW platform could achieve benefits similar to those obtained with a treadmill, but without using electric shock. Therefore, the proposed FNESRW platform can be used as an alternative system in clinical animal studies. The overall effect of the proposed platform was close to the treadmill group. Furthermore, recovery of hindlimbs after training on the proposed FNESRW was more rapid than for the control group. Most of the time, the running posture of the rats on the proposed platform involved the hindlimbs being higher than the forelimbs. Therefore, we believe that this running posture causes the hindlimbs of the rats to undergo heavier load training.

The advantage of the proposed system is the lack of interference from electric shocks and the stable training pace at high-intensity speeds. The protection against ischemic stroke that is provided by a treadmill is partly from the use of electrical stimuli (17). However, animals may exhibit a stress response to such stimuli, and this should be acknowledged in forced treadmill studies. Some studies have shown evidence of systemic stress, which may lead to adrenal hypertrophy, splenic atrophy, and increased circulating corticosterone, especially in male animals $(18,39)$. Therefore, any results that are obtained in studies using treadmills may not be completely caused by the exercise; instead, they may be partially caused by psychological stress. To exclude the interference of the electrical stimulus, this study proposed and implemented a wheel that does not use electric shock but provides the same benefits as a traditional treadmill in clinical trials.

The benefit of the variable speed movement patterntriggered neuroprotective factor against stroke is another relevant topic. Most animal experiments in the literature maintained $20 \mathrm{~m} / \mathrm{min}$ to $30 \mathrm{~m} / \mathrm{min}$ on a treadmill for $30-60 \mathrm{~min}$. The results showed that achieving $75 \%$ of maximum oxygen uptake $\left(\mathrm{VO}_{2} \max \right)$ enhanced neuroprotective factors, but it was unclear whether less than $60 \%$ of $\mathrm{VO}_{2} \mathrm{max}$ exercise also increased neuroprotective factors (40). Another advantage of the proposed system is that the number of revolutions and the revolution time of the wheel can be programmed. Therefore, the FNESRW exercise platform can be used to evaluate the neuroprotective benefit of running at various intensities and durations.

This study implemented an FNESRW system, which effectively trains rats in an automated mode for exercise preconditioning, thereby significantly reducing infarct volume after ischemic stroke. Unlike a traditional treadmill, 
the proposed platform forces a rat to continue highintensity running without using electric shock, resulting in experiments with less interference. Automated progressive training patterns were constructed to facilitate smooth running at high intensity. Moreover, in cooperation with automatic acceleration training patterns, the PVC runway wheel allows rats to run more steadily during highintensity training. The experimental data showed that the proposed FNESRW system for exercise preconditioning successfully provided a neuroprotective benefit of exercise for rats.

\section{References}

1. Cotman CW, Berchtold NC. Exercise: a behavioral intervention to enhance brain health and plasticity. Trends Neurosci 2002; 25: 295-301, doi: 10.1016/S0166-2236(02)02143-4.

2. Zogaib FG, Monte-Alto-Costa A. Moderate intensity physical training accelerates healing of full-thickness wounds in mice. Braz J Med Biol Res 2011; 44: 1025-1035, doi: 10.1590/S0100-879X2011007500115.

3. Pagnussat AS, Michaelsen SM, Achaval M, Ilha J, Hermel EE, Back FP, et al. Effect of skilled and unskilled training on nerve regeneration and functional recovery. Braz $\mathrm{J}$ Med Biol Res 2012; 45: 753-762, doi: 10.1590/S0100-879X2012007500084.

4. Chen YW, Chen SH, Chou W, Lo YM, Hung CH, Lin MT. Exercise pretraining protects against cerebral ischaemia induced by heat stroke in rats. $\mathrm{Br} J$ Sports Med 2007; 41: 597-602, doi: 10.1136/bjsm.2006.033829.

5. Ding $\mathrm{Y}$, Ding $\mathrm{YH}, \mathrm{Li} \mathrm{J}$, Rafols JA. Exercise induces integrin overexpression and improves neurovascular integrity in ischemic stroke. Stroke 2005; 36: 470, doi: 10.1161/01.STR. 0000166199.10017.c5.

6. Jin J, Kang HM, Park C. Voluntary exercise enhances survival and migration of neural progenitor cells after intracerebral haemorrhage in mice. Brain Inj 2010; 24: 533540, doi: 10.3109/02699051003610458.

7. Liebetanz D, Gerber J, Schiffner C, Schutze S, Klinker F, Jarry $\mathrm{H}$, et al. Pre-infection physical exercise decreases mortality and stimulates neurogenesis in bacterial meningitis. J Neuroinflammation 2012; 9: 168, doi: 10.1186/17422094-9-168

8. Wang RY, Yang YR, Yu SM. Protective effects of treadmill training on infarction in rats. Brain Res 2001; 922: 140-143, doi: 10.1016/S0006-8993(01)03154-7.

9. Li J, Luan X, Clark JC, Rafols JA, Ding Y. Neuroprotection against transient cerebral ischemia by exercise pre-conditioning in rats. Neurol Res 2004; 26: 404-408, doi: 10.1179/016164104225016038

10. Chang $\mathrm{CH}$, Kao $\mathrm{CH}$, Chio $\mathrm{CC}$, Lin $\mathrm{CH}$, Lin MT, Chang CP. Attenuating heatstroke-induced acute lung inflammation, edema, and injury in rats by exercise preconditioning. $J$ Trauma Acute Care Surg 2013; 74: 1052-1059, doi: 10.1097/ TA.0b013e31827a3618.

11. Kim G, Kim E. The effects of antecedent exercise on motor function recovery and brain-derived neurotrophic factor expression after focal cerebral ischemia in rats. J Phys Ther Sci 2013; 25: 553-556.

12. Park S, Kim dS, Kang $\mathrm{S}$. Exercise training attenuates

\section{Supplementary Material}

Click here to view [pdf].

\section{Acknowledgments}

The authors would like to thank Miss Ling-Yu Tang and Yu-Chieh Hsu from Chi-Mei Medical Center, Tainan, Taiwan, for their valuable assistance in testing the prototype system in real experiments with rats and comparing the results with those of other groups.

cerebral ischemic hyperglycemia by improving hepatic insulin signaling and beta-cell survival. Life Sci 2013; 93: 153-160, doi: 10.1016/j.lfs.2013.06.005.

13. Itoh $\mathrm{T}$, Imano $\mathrm{M}$, Nishida $\mathrm{S}$, Tsubaki M, Hashimoto $\mathrm{S}$, Ito $\mathrm{A}$, et al. Exercise inhibits neuronal apoptosis and improves cerebral function following rat traumatic brain injury. $J$ Neural Transm 2011; 118: 1263-1272, doi: 10.1007/s00702-0110629-2.

14. Hu X, Zheng $\mathrm{H}$, Yan $\mathrm{T}$, Pan $\mathrm{S}$, Fang J, Jiang $\mathrm{R}$, et al. Physical exercise induces expression of CD31 and facilitates neural function recovery in rats with focal cerebral infarction. Neurol Res 2010; 32: 397-402, doi: 10.1179/ $016164110 \times 12670144526309$

15. Ding YH, Ding Y, Li J, Bessert DA, Rafols JA. Exercise preconditioning strengthens brain microvascular integrity in a rat stroke model. Neurol Res 2006; 28: 184-189, doi: 10.1179/016164106X98053.

16. Kinni H, Guo M, Ding JY, Konakondla S, Dornbos D III, Tran R, et al. Cerebral metabolism after forced or voluntary physical exercise. Brain Res 2011; 1388: 48-55, doi: 10.1016/j.brainres.2011.02.076.

17. Hayes K, Sprague S, Guo M, Davis W, Friedman A, Kumar $A$, et al. Forced, not voluntary, exercise effectively induces neuroprotection in stroke. Acta Neuropathol 2008; 115: 289296, doi: 10.1007/s00401-008-0340-z.

18. Arida RM, Scorza CA, da Silva AV, Scorza FA, Cavalheiro EA. Differential effects of spontaneous versus forced exercise in rats on the staining of parvalbumin-positive neurons in the hippocampal formation. Neurosci Lett 2004; 364: 135-138, doi: 10.1016/j.neulet.2004.03.086.

19. Brown DA, Johnson MS, Armstrong CJ, Lynch JM, Caruso NM, Ehlers LB, et al. Short-term treadmill running in the rat: what kind of stressor is it? J Appl Physiol 2007; 103: 19791985, doi: 10.1152/japplphysiol.00706.2007.

20. Leasure JL, Jones M. Forced and voluntary exercise differentially affect brain and behavior. Neuroscience 2008; 156: 456-465, doi: 10.1016/j.neuroscience.2008.07.041.

21. Kennard JA, Woodruff-Pak DS. A comparison of low- and high-impact forced exercise: effects of training paradigm on learning and memory. Physiol Behav 2012; 106: 423-427, doi: 10.1016/j.physbeh.2012.02.023.

22. Caton SJ, Bielohuby M, Bai Y, Spangler LJ, Burget L, Pfluger $\mathrm{P}$, et al. Low-carbohydrate high-fat diets in combination with daily exercise in rats: effects on body weight regulation, body composition and exercise capacity. Physiol Behav 2012; 106: 
185-192, doi: 10.1016/j.physbeh.2012.02.003

23. Cao Y, Hu Y, Liu P, Zhao HX, Zhou XJ, Wei YM. Effects of a Chinese traditional formula Kai Xin San (KXS) on chronic fatigue syndrome mice induced by forced wheel running. J Ethnopharmacol 2012; 139: 19-25, doi: 10.1016/j.jep.2011. 08.030 .

24. Schmidt-Kassow M, Schadle $S$, Otterbein S, Thiel C, Doehring A, Lotsch J, et al. Kinetics of serum brain-derived neurotrophic factor following low-intensity versus highintensity exercise in men and women. Neuroreport 2012; 23: 889-893, doi: 10.1097/WNR.0b013e32835946ca.

25. Todoroki K, Sugiura T, Goto K, Naito H, Yoshioka T. Changes in heat shock protein induction in rat skeletal muscles following acute treadmill training at different speeds. J Phys Fit Sport 2004; 53: 537-547.

26. Symons JD, Hayashi Y, Ensunsa JL. Improved coronary vascular function evoked by high-intensity treadmill training is maintained in arteries exposed to ischemia and reperfusion. J Appl Physiol 2003; 95: 1638-1647.

27. Martins DF, Mazzardo-Martins L, Soldi F, Stramosk J, Piovezan AP, Santos AR. High-intensity swimming exercise reduces neuropathic pain in an animal model of complex regional pain syndrome type I: evidence for a role of the adenosinergic system. Neuroscience 2013; 234: 69-76, doi: 10.1016/j.neuroscience.2012.12.042.

28. Chang MW, Young MS, Lin MT. An inclined plane system with microcontroller to determine limb motor function of laboratory animals. J Neurosci Methods 2008; 168: 186194, doi: 10.1016/j.jneumeth.2007.09.013.

29. Chen J, Li Y, Wang L, Zhang Z, Lu D, Lu M, et al. Therapeutic benefit of intravenous administration of bone marrow stromal cells after cerebral ischemia in rats. Stroke 2001; 32: 1005-1011, doi: 10.1161/01.STR.32.4.1005.

30. Hung $\mathrm{CH}$, Chang NC, Cheng BC, Lin MT. Progressive exercise preconditioning protects against circulatory shock during experimental heatstroke. Shock 2005; 23: 426-433, doi: 10.1097/01.shk.0000159557.95285.96.

31. Demirel HA, Powers SK, Caillaud C, Coombes JS, Naito H, Fletcher LA, et al. Exercise training reduces myocardial lipid peroxidation following short-term ischemia-reperfusion. Med Sci Sports Exerc 1998; 30: 1211-1216, doi: 10.1097/ 00005768-199808000-00005.
32. Longa EZ, Weinstein PR, Carlson S, Cummins R. Reversible middle cerebral artery occlusion without craniectomy in rats. Stroke 1989; 20: 84-91, doi: 10.1161/01.STR.20.1.84.

33. Belayev L, Alonso OF, Busto R, Zhao W, Ginsberg MD. Middle cerebral artery occlusion in the rat by intraluminal suture. Neurological and pathological evaluation of an improved model. Stroke 1996; 27: 1616-1622, doi: 10.1161/ 01.STR.27.9.1616.

34. Gartshore G, Patterson J, Macrae IM. Influence of ischemia and reperfusion on the course of brain tissue swelling and blood-brain barrier permeability in a rodent model of transient focal cerebral ischemia. Exp Neurol 1997; 147: 353-360, doi: 10.1006/exnr.1997.6635.

35. Chen F, Suzuki $Y$, Nagai $N$, Jin L, Yu J, Wang $H$, et al. Rodent stroke induced by photochemical occlusion of proximal middle cerebral artery: evolution monitored with MR imaging and histopathology. Eur J Radiol 2007; 63: 6875, doi: 10.1016/j.ejrad.2007.01.005.

36. Bederson JB, Pitts LH, Germano SM, Nishimura MC, Davis $\mathrm{RL}$, Bartkowski HM. Evaluation of 2,3,5-triphenyltetrazolium chloride as a stain for detection and quantification of experimental cerebral infarction in rats. Stroke 1986; 17: 1304-1308, doi: 10.1161/01.STR.17.6.1304.

37. Ke Z, Yip SP, Li L, Zheng XX, Tong KY. The effects of voluntary, involuntary, and forced exercises on brainderived neurotrophic factor and motor function recovery: a rat brain ischemia model. PLoS One 2011; 6: e16643, doi: 10.1371 /journal.pone.0016643.

38. Jeneson JA, de Snoo MW, Verlinden NA, Joosten BJ, Doornenbal A, Schot $A$, et al. Treadmill but not wheel running improves fatigue resistance of isolated extensor digitorum longus muscle in mice. Acta Physiol 2007; 190: 151-161, doi: 10.1111/j.1748-1716.2007.01680.x.

39. Moraska A, Deak T, Spencer RL, Roth D, Fleshner M. Treadmill running produces both positive and negative physiological adaptations in Sprague-Dawley rats. $A m \mathrm{~J}$ Physiol Regul Integr Comp Physiol 2000; 279: R1321R1329.

40. Starnes JW, Taylor RP, Ciccolo JT. Habitual low-intensity exercise does not protect against myocardial dysfunction after ischemia in rats. Eur J Cardiovasc Prev Rehabil 2005; 12: $169-174$ 